\title{
6p22.3 deletion: report of a patient with autism, severe intellectual disability and electroencephalographic anomalies
}

\author{
Daniela Di Benedetto ${ }^{*^{*}}$, Giuseppa Di Vita ${ }^{4 \dagger}$, Corrado Romano ${ }^{2}$, Mariangela Lo Giudice ${ }^{3}$, Girolamo Aurelio Vitello ${ }^{4}$, \\ Marinella Zingale ${ }^{5}$, Lucia Grillo ${ }^{1}$, Lucia Castiglia', Sebastiano Antonino Musumeci ${ }^{4}$ and Marco Fichera, ${ }^{1,6}$
}

\begin{abstract}
Background: The interstitial $6 p$ deletions, involving the 6p22-p24 chromosomal region, are rare events characterized by variable phenotypes and no clear genotype-phenotype correlation has been established so far.

Results: High resolution array-CGH identified $1 \mathrm{Mb}$ de novo interstitial deletion in 6p22.3 chromosomal region in a patient affected by severe Intellectual Disability (ID), Autism Spectrum Disorders (ASDs), and electroencephalographic anomalies. This deletion includes ATXN1, DTNBP1, JARID2 and MYLIP genes, known to play an important role in the brain, and the GMPR gene whose function in the nervous system is unknown.

Conclusions: We support the suggestion that ATXN1, DTNBP1, JARID2 and MYLIP are candidate genes for the pathophysiology of ASDs and ID, and we propose that deletion of DTNBP1 and/or JARID2 contributes to the hypotonia phenotype.
\end{abstract}

Keywords: 6p22.3 deletion, Array-CGH, ASDs, Hypotonia, DTNBP1

\section{Background}

The interstitial deletions involving the 6p22-p24 chromosomal region are characterized by variable phenotype, according to the different size of the deleted regions and the small amount of patients reported to date. The clinical phenotype includes psychomotor delay, hypotonia, defects in brain, heart, kidney and eye, short neck, craniofacial malformations, as well as clinodactyly or syndactyly [1-4]. Recently, six patients carrying overlapping deletions in the 6p22.3-p24.3 region were described. They presented common features of $6 p$ deletion syndrome, but also neurological or behavioral abnormalities, including speech delay, autism spectrum disorders (ASDs), hyperactivity/ADHD, or other behavioral abnormalities [5]. Here, we report a $1 \mathrm{Mb}$ interstitial deletion in the $6 \mathrm{p} 22.3$ chromosomal region identified by 60 -mer oligonucleotide $180 \mathrm{~K}$ array comparative genomic hybridization (array-CGH) in an 18 years-old

\footnotetext{
* Correspondence: ddibenedetto@oasi.en.it

${ }^{\dagger}$ Equal contributors

${ }^{1}$ Laboratory of Medical Genetics, I.R.C.C.S. Associazione Oasi Maria Santissima, Troina, Italy

Full list of author information is available at the end of the article
}

patient affected by severe ID, ASDs and electroencephalographic (EEG) anomalies. Further analysis demonstrated that the deletion was de novo and microsatellite segregation analysis revealed that the rearrangement involved the paternal chromosome 6. Four genes (ATXN1, DTNBP1, JARID2 and MYLIP ) located in the deleted 6p22.3 region are involved in the development and function of the nervous system [4]. Our finding supports the contributory effect of these genes in the pathophysiology of ASDs and ID $[4,5]$. Furthermore, we propose a possible involvement of DTNBP1 and/or JARID2 genes in hypotonia.

\section{Clinical description}

The patient is the first of two siblings born to healthy non-consanguineous parents. The pregnancy was characterized by weak fetal movements and he was born at term by induced vaginal delivery. His birth weight was $4250 \mathrm{~g}\left(>97^{\text {th }}\right.$ centile) and he showed physiological jaundice, treated by phototherapy. Dacryocystitis by lacrimal duct stenosis, bilateral hydrocele, umbilical hernia and left lateral xiphoid epigastric hernia were observed. 
The patient had delayed psychomotor development, he walked at 15 months and later he showed autonomous deambulations with awkward movements. He presented with delayed speech. We first observed the patient at the age of 3 years and 9 months. The patient presented with long face, drooling, epicanthic folds, macrosomia (head circumference, height and weight $>97^{\text {th }}$ centile), central obesity, large testes, knock knees and bilateral valgus flat foot. Diffuse ligament laxity and muscular hypotonia, but with standard tropism and no deficit of strength, where also present. When observed at the age of 12 years and 7 months, the patient showed normal deep tendon reflexes, shifting with age to hyporeflexia, especially at lower limbs. He had poor fine motor skills and general coordination. His gait was broad-based with clampsy movements.

The neurological examination, performed at the age of 3 years and 9 months, showed severe ID and typical autistic traits, impairments in environmental and social interactions, avoidance of spontaneous or requested eye contact, very limited verbal and non-verbal communication, limited interests, and repetitive behaviors with frequent hand flapping, head and trunk rocking. This behavioral phenotype was classified in the autism spectrum according to ADOS-G and ADI-R testing. The patient was clearly hyperactive and presented with behavioral problems including self-injury (head banging) and, less frequently, aggressiveness (biting) towards others. He suffered from sleep disorders.

Magnetic resonance (MR) imaging performed at the age of 4 years and 6 months revealed mild abnormalities in the posterior periventricular white matter and enlarged sulci and ventricles. EEG showed paroxysmal discharges, such as spikes and spike-and-wave complexes, isolated or in short sequences that occurred in the central and temporal regions of both hemispheres, significantly activated during sleep.

Electromyography (EMG) showed an increased number of polyphasic low-amplitude potentials (the patient did not cooperate for electroneurography).

Heart and kidney ultrasounds were normal (asymptomatic kidney microlithiasis). Metabolic screening and fragile $\mathrm{X}$ testing were normal. Conventional cytogenetic studies showed a normal male karyotype (46,XY).

\section{Results}

We have identified in our patient a de novo interstitial deletion of $1 \mathrm{Mb}$ in the $6 \mathrm{p} 22.3$ chromosomal region as described in Figure 1. No other significant $\mathrm{CNV}$ was detected. The deletion involved five genes ATXN1, DTNBP1, JARID2, MYLIP and GMPR. The distal breakpoint was located in intron 1 of JARID2 gene, between genomic position 15.28 and $15.29 \mathrm{Mb}$, while the proximal breakpoint was located in intron 7 of $A T X N 1$ gene, between the genomic position 16.36 and $16.37 \mathrm{Mb}$ (according to human genome assembly, February 2009, hg19, http://genome.ucsc.edu/). Minimal and maximal sizes of deletion were $1.07 \mathrm{Mb}$ and $1.09 \mathrm{Mb}$, respectively. This aberration was not reported in the online Database of Genomic Variants, a curated collection of structural variations identified in healthy control samples (http:// projects.tcag.ca/variation/?source=hg19), and was not present in the parental samples investigated by arrayCGH. Further microsatellite segregation analysis with markers located in the deleted segment, confirmed the deletion and demonstrated a paternal origin of this rearrangement (data not shown).

\section{Discussion}

The interstitial deletions of $6 \mathrm{p}$ are characterized by variable sizes and phenotypes. A minimal critical region (MCR) for the $6 \mathrm{p} 22$ deletion phenotype has been proposed by Bremer et al. [4] who identified a $2.2 \mathrm{Mb}$ region involving 12 genes. Some of them were proposed as candidate genes, but none has been strongly associated with specific clinical features of the syndrome. Here we report a $6 \mathrm{p}$ deletion of about 1 $\mathrm{Mb}$ encompassing five genes (ATXN1, DTNBP1, JARID2, $M Y L I P$ and GMPR), identified in a patient with severe ID, hypotonia, brain anomalies, EEG abnormalities, macrosomia, obesity, and ASDs with associated hyperactivity and behavioral abnormalities. In Figure 2 we present a genotype-phenotype correlation between our patient and others reported in the literature [1-6] and we observe that the phenotype of our patient overlaps with previously reported cases of $6 \mathrm{p} 22.3$ deletions, particularly for ID, ASDs and hypotonia. Our findings support the suggestion [4,5], that ATXN1, DTNBP1, JARID2 and MYLIP are candidate genes for ID and ASDs. Furthermore, we suggest that two deleted genes (JARID2 and/or DTNBP1) can be involved in hypotonia.

The deletion of ATXN1 gene could be associated to ID and ASDs. The ataxin-1 protein is localized in the neurons of the basal ganglia, pons, cortex and Purkinje cells of the cerebellum and it is involved in the regulation of transcription and RNA processing. ATXN1 is the cause of spino-cerebellar ataxia type 1 (SCA1, OMIM:164400), a dominantly inherited neurodegenerative disease, through CAG repeat expansion with a gain-of-function mechanism [7], while loss-of-function of ATXN1 seems to lead to motor coordination impairments and learning deficits [8]. Researchers have found a significant association between SNPs in the gene and intelligence quotient in ADHD patients [9] and also a correlation among loss-of-function of ATXN1 and increased amyloid $\beta$-protein levels in Alzheimer patients [10].

JARID2 gene is a DNA-binding transcriptional repressor. It is expressed in embryonic and adult human neurons and it is a critical factor for the cardiovascular development 


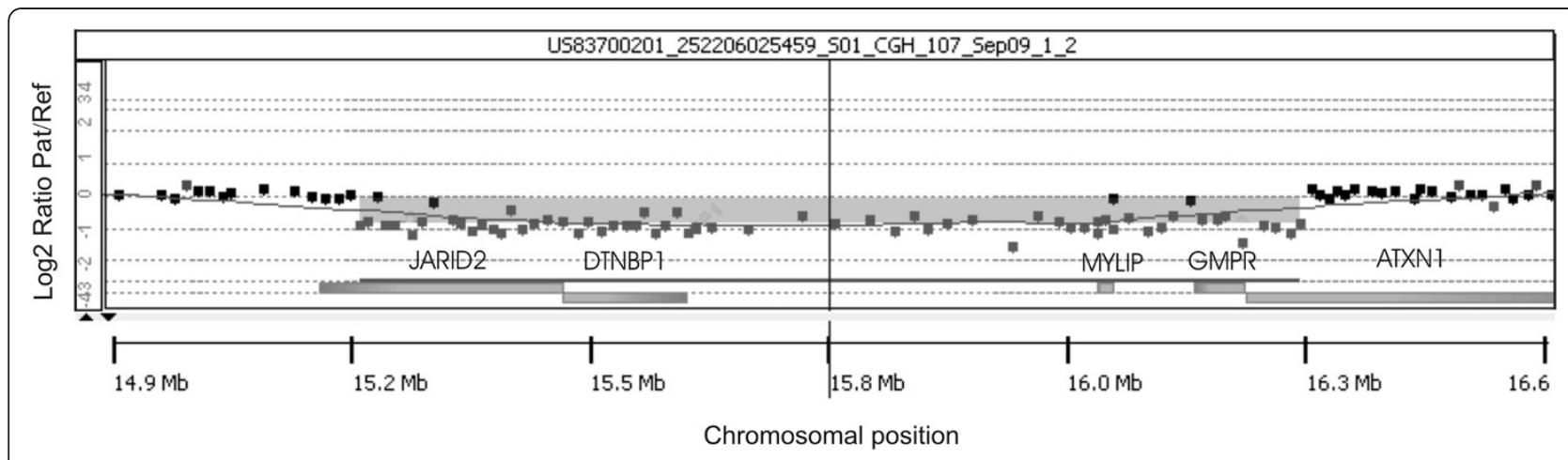

Figure 1 Array-CGH deletion. Detailed view of 6p22.3 segment with in the Y-axis the log2 signal ratio of chromosome 6 probes (patient /reference) plotted as a function of chromosomal position (X-axis). The shaded region represents the $1 \mathrm{Mb}$ deletion identified in our patient.

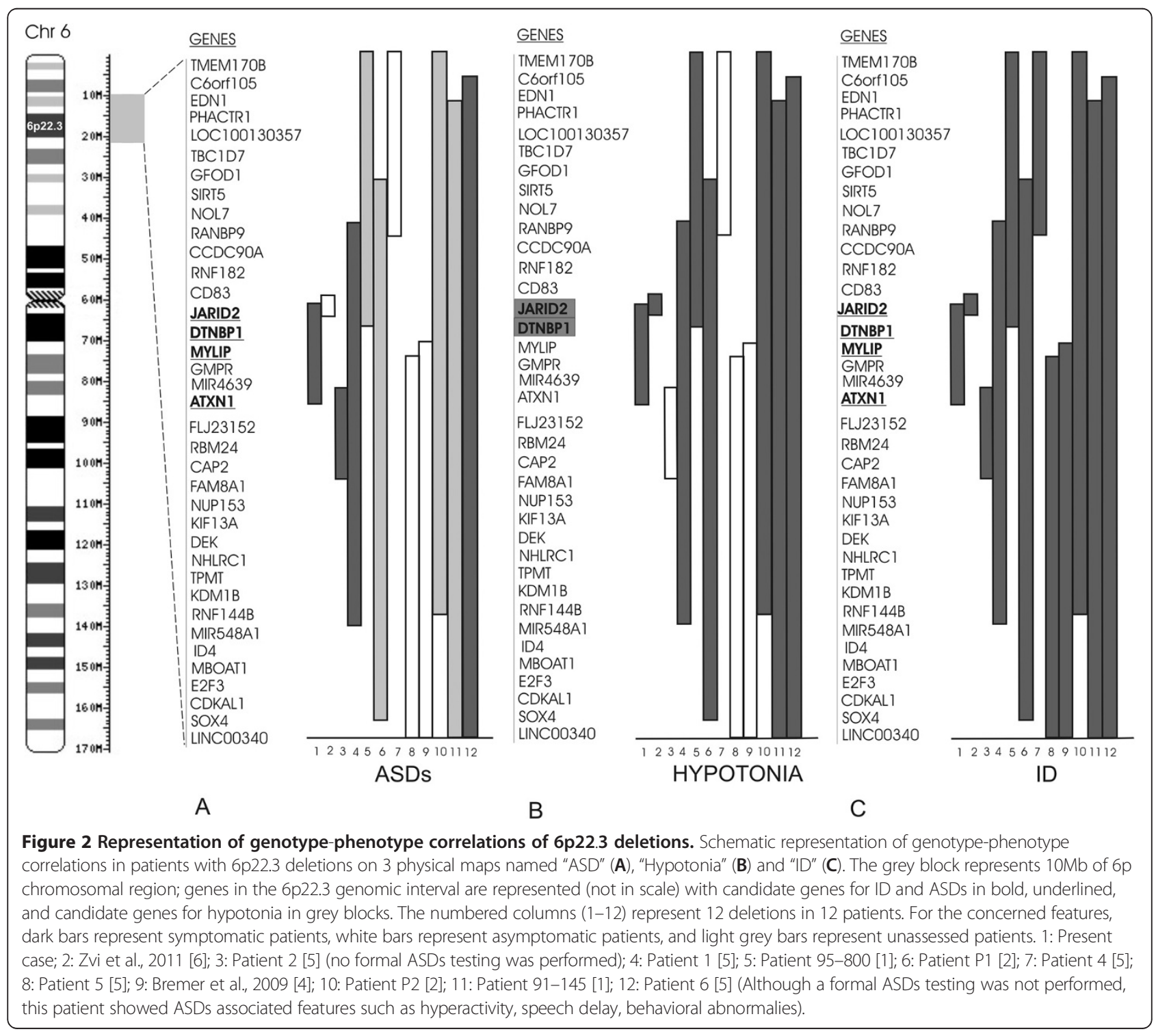


[11,12]. Furthermore, a significant association between a SNP (rs7766973) in the JARID2 gene and autism has been reported [13].

The myosin regulatory light-chain interacting protein (MYLIP) is a novel member of the ezrin-radixin-moesin protein family (ERM). These proteins participate in the regulation of cell proliferation, differentiation, and signals transduction [14]. Olsson et al. [15] found that MYLYP is expressed in rat embryonic hippocampal neuronal cell bodies and growth cones, where it works as a neurite outgrowth suppressor [16]. MYLIP is also known as IDOL, inducible degrader of the LDL receptor, based on its involvement in cholesterol regulation [17]. IDOL knockdown in hepatocytes increases LDLR protein levels and promotes LDL uptake through LXR-IDOL-LDLR pathway [18]. The impairment of this function can be related to the obesity observed in our patient.

$D T N B P 1$ is a gene with several important functions. The encoded protein interacts with $\alpha$ - and $\beta$-dystrobrevins as component of the dystrophin protein complex (DPC) in muscle and brain, mediating the link between the DPC and intracellular signaling cascade. In muscle, it functions as a structural scaffold linking the $\alpha$-dystrobrevins to sarcolemma [19] and mutations which disrupt the DPC can give rise to numerous types of skeletal myopathy, including Duchenne muscular dystrophy (DMD [MIM 310200]) in humans and mice [20]. In the mouse brain, dysbindin is enriched in regions implicated in synaptic structure and signaling of the glutamatergic neuronal system [19]. Numakawa et al. [21] suggest that dysbindin might influence exocytotic glutamate release via upregulation of the molecules in pre-synaptic machinery as synaptosomal-associated protein (SNAP25) and synapsin 1 (SYN1). These results suggest that dysbindin might be one of the regulatory proteins in the excitatory neurotransmission and can be associated also with EEG anomalies observed in our patient.

Moreover, DTNBP1 has been connected with autistic features observed sometimes in patients with Duchenne muscular dystrophy. Hendriksen et al. [22] reported in a population of males with Duchenne muscular dystrophy a neuropsychiatric comorbidity, including increased frequencies of ASDs and ADHD.

The Guanosine monophosphate reductase (GMPR) catalyzes the irreversible NADPH-dependent reductive deamination of guanosine monophosphate (GMP) to inosine monophosphate (IMP) and is involved in thermogenesis [23], but its function in the nervous system is unknown.

\section{Conclusions}

Using array-CGH, we identified $1 \mathrm{Mb}$ deletion in $6 \mathrm{p} 22.3$ in a male patient with ASDs, ID, and EEG anomalies and we delineate a genotype-phenotype correlation for 6p22.3 deleted patients. We suggest that JARID2 and/or $D T N B P 1$ genes can be candidate for hypotonia and we support the role of ATXN1, DTNBP1, JARID2 and MYLIP genes in the pathophysiology of ASDs and ID $[4,5]$. Incomplete penetrance of ASDs, as shown in Figure $2 \mathrm{~A}$, may be due to other genetic, epigenetic or environmental factors.

Additional clinically and molecularly well-defined, patients will be required to improve the genotypephenotype correlation of the 6p22-p24 deletion syndrome.

\section{Methods}

\section{Consents}

The study was approved by the local ethics committee on 18 May 2012 and written informed consent was obtained for the patient for publication of this report.

Genomic DNAs were extracted by standard procedures, and tested for submicroscopic copy number variants using the commercially available Human Genome array-CGH 180K Microarray (Agilent Technologies, Palo Alto, CA), which provides an average resolution of around $13 \mathrm{~Kb}$. The patient DNA was matched versus a male reference DNA (NA10851, Coriell Institute), and the assay performed according to the manufacturer's protocol version 7.1. Arrays were scanned using the Agilent Microarray Scanner. Data were extracted using Feature Extraction Software version 10.7 and analyzed using Agilent Genomic Workbench Lite Edition 6.5. The parental samples were investigated with $180 \mathrm{~K}$ array-CGH platform, and parental origin was tested with microsatellite analysis of an existing polymorphic marker (D6S260) and two new markers developed in this study (JAR1, forward primer $5^{\prime}$-GCCTT CTTTAACCGCTTGCA-3', reverse primer 5'-CCTCATG GTTACTGCATCCTGTAA-3', and JAR2 forward primer 5'-CTGCTATGGATTCATGGCCG-3', reverse primer 5'-CCTGCCTTCCAAATGTGTTATACA- $3^{\prime}$ ) all located inside the JARID2 gene.

The PCR products were electrophoresed on a ABI 310 Genetic Analyser (Applied Biosystems) and analyzed by Genescan software.

\footnotetext{
Abbreviations

ID: Intellectual disability; ASDs: Autism spectrum disorders; ADHD: Attention deficit hyperactive disorders; EEG: Electroencephalography; MRI: Magnetic resonance imaging; EMG: Electromyography; CGH: Comparative genomic hybridization; MCR: Minimal critical region; DPC: Dystrophin protein complex; ERM: Ezrin-radixin-moesin protein family; DMD: Duchenne muscular dystrophy.
}

\section{Competing interests}

The authors declare that they have no competing interests.

Authors' contributions

DDB and PDV designed the experiment and drafted the manuscript. LG, LC and DDB performed the chromosomal microarray assay and data analysis. MLG performed the microsatellite analysis. MZ performed the ADOS-G and ADI-R tests. CR, PDV and SAM examined clinically the patient. GAV 
performed the EMG analysis. MF drafted the manuscript. All authors read and approved the final manuscript.

\section{Aknowledgments}

This study was supported by Telethon (grant no. GGP08226 to M.F.).

\section{Author details}

'Laboratory of Medical Genetics, I.R.C.C.S. Associazione Oasi Maria Santissima, Troina, Italy. ${ }^{2}$ Unit of Pediatrics and Medical Genetics, I.R.C.C.S. Associazione Oasi Maria Santissima, Troina, Italy. ${ }^{3}$ Unit of Neuromuscular Disease, I.R.C.C.S. Associazione Oasi Maria Santissima, Troina, Italy. ${ }^{4}$ Unit of Neurology, I.R.C.C.S. Associazione Oasi Maria Santissima, Troina, Italy. ${ }^{5}$ Unit of Psychology, I.R.C.C.S. Associazione Oasi Maria Santissima, Troina, Italy. ${ }^{6}$ Medical Genetics, University of Catania, Catania, Italy.

Received: 12 November 2012 Accepted: 6 December 2012 Published: 17 January 2013

\section{References}

1. Davies AF, Mirza G, Sekhon G, Turnpenny P, Leroy F, Speleman F, Law C, van Regemorter N, Vamos E, Flinter F, Ragoussis J: Delineation of two distinct $6 p$ deletion syndromes. Hum Genet 1999, 104:64-72.

2. Davies AF, Olavesen MG, Stephens RJ, Davidson R, Delneste D, van Regemorter N, Vamos E, Flinter F, Abusaad I, Ragoussis J: A detailed investigation of two cases exhibiting characteristics of the $6 p$ deletion syndrome. Hum Genet 1996, 98:454-459.

3. van Swaay E, Beverstock GC, van de Kamp JJ: A patient with an interstitial deletion of the short arm of chromosome 6. Clin Genet 1988, 33:95-101.

4. Bremer A, Schoumans J, Nordenskjöld M, Anderlid BM, Giacobini M: An interstitial deletion of $7.1 \mathrm{Mb}$ in chromosome band $6 \mathrm{p} 22.3$ associated with developmental delay and dysmorphic features including heart defects, short neck, and eye abnormalities. Eur J Med Genet 2009, 52:358-362.

5. Celestino-Soper PBS, Skinner C, Schroer R, Eng P, Shenai J, Nowaczyk MMJ, Terespolsky D, Cushing D, Patel GS, Immken LD, Willis A, Wiszniewska J, Matalon R, Rosenfeld JA, Stevenson RE, Kang SHL, Cheung SW, Beaudet AL, Stankiewicz P: Deletions in chromosome 6p22.3-p24.3, including ATXN1, are associated with developmental delay and autism spectrum disorders. Mol Cytogenet 2012, 5:5-17.

6. Zvi T, Yaron Y, Orr-Urtreger A, Ben-Shachar S: JARID2 isolated deletion is associated with velo-palatal insufficiency, hypotonia and developmental delay, Abstract/Program 955T. Montreal, Canada: Presented at the 12th International Congress of Human Genetics/61st Annual Meeting of The American Society of Human Genetics; October 13, 2011.

7. Donato SD, Mariotti C, Taroni F: Spinocerebellar ataxia type 1. Handb Clin Neurol 2012, 103:399-421.

8. Matilla A, Roberson ED, Banfi S, Morales J, Armstrong DL, Burright EN, Orr $H T$, Sweatt JD, Zoghbi HY, Matzuk MM: Mice lacking ataxin-1 display learning deficits and decreased hippocampal paired-pulse facilitation. J Neurosci 1998, 18:5508-5516.

9. Rizzi TS, Arias-Vasquez A, Rommelse N, Kuntsi J, Anney R, Asherson P, Buitelaar J, Banaschewski T, Ebstein R, Ruano D, Van der Sluis S, Markunas CA, Garrett ME, Ashley-Koch AE, Kollins SH, Anastopoulos AD, Hansell NK, Wright MJ, Montgomery GW, Martin NG, Harris SE, Davies G, Tenesa A, Porteous DJ, Starr JM, Deary IJ, St Pourcain B, Davey Smith G, Timpson NJ, Evans DM, et al: The ATXN and TRIM3 genes are related to intelligence in an ADHD background: evidence from a large collaborative study totaling totaling 4,963 subjects. Am J Med Genet B Neuropsychiatr Genet 2011, 156:145-157.

10. Zhang C, Browne A, Child D, Divito JR, Stevenson JA, Tanzi RE: Loss of function of ATXN1 increases amyloid beta-protein levels by potentiating beta-secretase processing of beta-amyloid precursor protein. $J$ Biol Chem 2010, 285:8515-8526.

11. Berge-Lefranc JL, Jay P, Massacrier A, Cau P, Mattei MG, Bauer S, Marsollier C, Berta P, Fontes M: Characterization of the human jumonji gene. Hum Mol Genet 1996, 5:1637-1641.

12. Kim TG, Kraus JC, Chen J, Lee Y: JUMONJI, a critical factor for cardiac development, functions as a transcriptional repressor. J Biol Chem 2003, 2478:42247-42255.

13. Carayol J, Schellenberg GD, Dombroski B, Genin E, Rousseau F, Dawson G: Autism risk assessment in siblings of affected children using sex-specific genetic scores. Mol Autism 2011, 21:2-17.
14. Bornhauser BC, Johansson C, Lindholm D: Functional activities and cellular localization of the ezrin, radixin, moesin (ERM) and RING zinc finger domains in MIR. FEBS Lett 2003, 553:195-199.

15. Olsson PA, Bornhauser BC, Korhonen L, Lindholm D: Neuronal expression of the ERM-like protein MIR in rat brain and its localization to human chromosome 6. Biochem Biophys Res Commun 2000, 279:879-883.

16. Olsson P-A, Korhonen L, Mercer EA, Lindholm D: MIR is a novel ERM-like protein that interacts with myosin regulatory light chain and inhibits neurite outgrowth. J Biol Chem 1999, 274:36288-36292.

17. Lindholm D, Bornhauser BC, Korhonen L: Mylip makes an Idol turn into regulation of LDL receptor. Cell Mol Life Sci 2009, 66:3399-3402.

18. Zelcer N, Hong C, Boyadjian R, Tontonoz P: LXR regulates cholesterol uptake through Idol-dependent ubiquitination of the LDL receptor. Science 2009, 325:100-104.

19. Benson MA, Newey SE, Martin-Rendon E, Hawkes R, Blake DJ: Dysbindin, a novel coiled-coil-containing protein that interacts with the dystrobrevins in muscle and brain. J Biol Chem 2001, 276:24232-24241.

20. Bushby KM: The limb-girdle muscular dystrophies-multiple genes, multiple mechanisms. Hum Mol Genet 1999, 8:1875-1882.

21. Numakawa T, Yagasaki Y, Ishimoto T, Okada T, Suzuki T, Iwata N, Ozaki N, Taguchi T, Tatsumi M, Kamijima K, Straub RE, Weinberger DR, Kunugi H, Hashimoto R: Evidence of novel neuronal functions of dysbindin, a susceptibility gene for schizophrenia. Hum Mol Genet 2004, 13:2699-2708.

22. Hendriksen JG, Vles JS: Neuropsychiatric disorders in males with duchenne muscular dystrophy: frequency rate of attention-deficit hyperactivity disorder (ADHD), autism spectrum disorder, and obsessive-compulsive disorder. J Child Neurol 2008, 23:477-481.

23. Salvatore D, Bartha T, Larsen PR: The guanosine monophosphate reductase gene is conserved in rats and its expression increases rapidly in brown adipose tissue during cold exposure. J Biol Chem 1998, 273:31092-31096

doi:10.1186/1755-8166-6-4

Cite this article as: Di Benedetto et al:: 6 p22.3 deletion: report of a patient with autism, severe intellectual disability and electroencephalographic anomalies. Molecular Cytogenetics 2013 6:4.

\section{Submit your next manuscript to BioMed Central and take full advantage of:}

- Convenient online submission

- Thorough peer review

- No space constraints or color figure charges

- Immediate publication on acceptance

- Inclusion in PubMed, CAS, Scopus and Google Scholar

- Research which is freely available for redistribution 